Kairos. Journal of Philosophy \& Science 22, 2019

Center for the Philosophy of Sciences of Lisbon University

\title{
From Effect to Cause: Deductive Reasoning
}

Ricardo Tavares da Silva

Faculdade de Letras da Universidade de Lisboa ricardo.silva@campus.ul.pt

DOI 10.2478/kjps-2019-0011

\begin{abstract}
According to the traditional view, the following incompatibility holds true: in reasoning, either there is warrant (certainty) or there is novelty. If there is warrant, there is not novelty: that would be the case of deductive reasoning. If there is novelty, there is not warrant: that would be the case of inductive reasoning. Causal reasoning would belong to the second group because there is novelty and, therefore, there is not warrant in it. I argue that this is false: reasoning may have novelty and, nevertheless, be a deductive one. That is precisely what happens in (some) causal reasoning. And I will develop the following line of argumentation: one thing is to warrant that some state of affairs exists and other thing is to warrant that warrant. So we may have correct deductive reasoning without having certainty of that correction, like in some cases of causal reasoning.
\end{abstract}

Keywords: Causal Reasoning; Deductive Reasoning; Synthetic Deductive Reasoning; Kant; Hume.

\section{Causal Reasoning: Inductive Reasoning?}

Philosophical tradition has accepted that causal reasoning is a kind of inductive reasoning. For instance, Copi, Cohen and McMahon, in their paper Causal Reasoning, say that:

"Induction goes far beyond analogical arguments [the last kind of inductive reasoning they analyzed]. (...) When we 
know, or think we know, that one thing is the cause of another, or the effect of another, we can reason from cause to effect, or from effect to cause. (...) If the supposed relations between cause and effect have been correctly established, the reasoning based on those relations is very powerful".'

Even more explicitly is their definition of causal reasoning:

"Inductive reasoning in which some effect is inferred from what is assumed to be its cause, or some cause is inferred from what is assumed to be its effect". ${ }^{2}$

But soon after, what seems to me to be a puzzling statement is made:

"Causal reasoning is also of the very greatest practical importance. Our ability to control our environment, to live successfully and to achieve our purposes, depends critically on our knowledge of causal connections. To cure some disease, for example, physicians must know its cause - and of course they must learn the effects (including the side effects) of the drugs they administer". ${ }^{3}$

What is striking in this set of sentences is the fact that, now, the authors are not talking about causal reasoning; they are talking about the knowledge of causal connections, from which causal reasoning is made. But, previously, the authors had distinguished between the correct establishment of the supposed relations between cause and effect and the reasoning based on those relations: being so, why are they talking about those relations instead of talking about causal reasoning itself?

And why does this matter? Because the rest of their paper is devoted to the evaluation of methods of discovery/justification of causal relations and, at least since Hume, these methods are considered to be inductive. Hence, one is led to think that causal reasoning is inductive too. But this

1 Copi, Cohen and McMahon, 2014, 514.

2 Copi, Cohen and McMahon, 2014, 514.

3 Copi, Cohen and McMahon, 2014, 514. 
is a mistake: the establishment of causal relationships can be inductive without the inferences based on these relations being inductive too.

The same perplexity is motivated by Hume's words. In the Enquiry, Hume begins by saying that:

"All reasonings concerning matter of fact seem to be founded on the relation of Cause and Effect. By means of that relation alone we can go beyond the evidence of our memory and senses. If you were to ask a man, why he believes any matter of fact, which is absent; for instance, that his friend is in the country, or in France; he would give you a reason; and this reason would be some other fact; as a letter received from him, or the knowledge of his former resolutions and promises. A man, finding a watch or any other machine in a desert island, would conclude, that there had once been men in that island. All our reasonings concerning fact are of the same nature. And here it is constantly supposed, that there is a connection between the present fact and that which is inferred from it. Were there nothing to bind them together, the inference would be entirely precarious. The hearing of an articulate voice and rational discourse in the dark assures us of the presence of some person: Why? Because these are the effects of the human make and fabric, and closely connected with it. If we anatomize all the other reasonings of this nature, we shall find that they are founded on the relation of cause and effect (...)". ${ }^{4}$

Ahead Hume says that:

"If we would satisfy ourselves, therefore, concerning the nature of that evidence, which assures us of matters of fact, we must enquire how we arrive at the knowledge of cause and effect. I shall venture to affirm, as a general proposition, which admits of no exception, that the knowledge of

$4 \quad$ Hume, 2007, Sec. IV, 19. 
this relation is not, in any instance, attained by reasonings a priori; but arises entirely from experience, when we find, that any particular objects are constantly conjoined with each other". ${ }^{5}$

Hume begins by referring to the inference from effects to causes and then refers to the knowledge of the causal relation itself. The confusion between the two operations - from effect to cause and from some fact to a causal connection - becomes even more evident in passages such as the following:

"Necessity may be defined two ways, conformably to the two definitions of cause, of which it makes an essential part. It consists either in the constant conjunction of like objects, or in the inference of the understanding from one object to another. Now necessity, in both these senses, (which, indeed, are, at bottom, the same) has universally, though tacitly, in the schools, in the pulpit, and in common life, been allowed to belong to the will of man; and no one has ever pretended to deny, that we can draw inferences concerning human actions, and that those inferences are founded on the experienced union of like actions, with like motives, inclinations, and circumstances". ${ }^{6}$

If, on the one hand, Hume sees the psychological association between two events as the source of the notion of causality, on the other hand, to him there is an inference from one to another, and an inference is not a psychological association, namely, the psychological association that originates causality in our minds. This confusion between inferring causes and inferring causal relations - and therefore between discovering the explicans and discovering the explanatory relation itself (the word 'explanation' is ambiguous) - is the (or one sufficient) source of a principle implicit and that have conditioned the entire investigation made in Logic: in an inference, either i) there is warrant (i.e., the inference is

$5 \quad$ Hume, 2007, Sec. IV, 19.

6 Hume, 2007, Sec. VIII, 70. 
a deductive one), and then the inference is a analytic one (i.e., without novelty), or ii) there is novelty (i.e., the inference is a synthetic one), and then the inference is an inductive one (i.e., without warrant). Since in causal reasoning there is novelty, then it must be of the inductive kind.

I think that this incompatibility principle is wrong. However, he holds true for two species of reasoning: analytic deduction - the traditional deduction - and synthetic induction - called abduction by Charles Peirce. More specifically, traditional deduction has had an almost unanimous acceptance among logicians and philosophers, who consider the incompatibility principle to be true, because of its undeniable validity regard to that type of reasoning.

Besides the incompatibility principle, another principle, the negative incompatibility principle, occupies a firm place in the history of Logic: in an inference, either i) there isn't warrant, and then the inference is a synthetic one, or ii) there isn't novelty, and then the inference is a deductive one. This principle holds true too for analytic deduction and synthetic induction, but nothing more.

\section{Reasoning and Meaning}

Let's assume the following definition of valid deductive reasoning: the truth of the conclusion derives with certainty (or warrant) from the truth of the premise(s). But why? What kind of relation bears between premise(s) and conclusion? If it's not a truth-functional one (if it 's intrinsic to the premise and the conclusion, aside their truth values), what is it? ${ }^{7}$

$7 \quad$ Truth-functionality is being put aside because of the so called 'paradoxes of the material implication'. Russell's material implication (' $p$ implies $q$ ' means 'Either $p$ is false or $q$ is true'; see Russell, 1997, 94) allows, for example, anything to be inferred from a falsehood, encompassing cases that we intuitively do not consider to be of logical validity. C.I. Lewis' strict implication (' $p$ implies $q$ ' means 'It is not possible for $p$ to be true and $q$ to be false'; see Lewis, 1918, 293) allows, for example, anything to be inferred from a necessary falsehood, encompassing too cases that we intuitively do not consider to be of logical validity. It should be noted that the so called 'strict implication' is a necessary material implication: in the words of von Wright, "one proposition strictly implies another proposition, if the (material) implication of the second by the first is necessary" (von Wright, 1951, 9). 
To answer that, let's look at a couple of examples of valid deductive reasoning according to the tradition:
A. All men are mortal
$\therefore$ Socrates (who is a man) is mortal.
B. $x$ is (a case of) propositional knowledge
$\therefore x$ is a belief.

Let us call the first type of reasoning 'formal deduction' and the second one 'material deduction' (not to be confused with the truth-functional type). In both cases one should ask: what makes the conclusion to be validly derived from the premise? If we substitute the sentences contained in the premises for what they mean, we'll get an answer:
A. All men are mortal $=_{\text {mean }}$ Parmenides (who is a man) is mortal and Pythagoras (who is a man) is mortal and Socrates (who is a man) is mortal and Plato (who is a man) is mortal and etc.
B. $x$ is (a case of) propositional knowledge $=_{\text {mean }} x$ is a belief $+x$ is true + etc $^{8}$

'Etc.' appears instead of what's missing in order for the meaning of the sentences to be complete (whatever is missing). In both examples, the conclusion is already contained in the premise: 'Socrates (who is a man) is mortal' is already included in the meaning of 'All men are mortal' and ' $x$ is a belief' is already included in the meaning of ' $x$ is (a case of) propositional knowledge'. The conclusion says the same as the premise (even if partially).

Such fact is well known by philosophers. For instance, Stuart Mill, in A System of Logic - Ratiocinative and Inductive, asserts that:

"We have now to inquire, whether the syllogistic process, that of reasoning from generals to particulars, is, or is not, a process of inference; a progress from the known to the unknown; a means of coming to a knowledge of something which we did not know before.

$8 \quad$ Assuming that the tripartite definition of propositional knowledge is the correct one. 
Logicians have been remarkably unanimous in their mode of answering this question. It is universally allowed that a syllogism is vicious if there be anything more in the conclusion than was assumed in the premises.

But this is, in fact, to say, that nothing ever was, or can be, proved by syllogism, which was not known, or assumed to be known, before. Is ratiocination, then, not a process of inference? And is the syllogism, to which the word reasoning has so often been represented to be exclusively appropriate, not really entitled to be called reasoning at all?

This seems an inevitable consequence of the doctrine, admitted by all writers on the subject, that a syllogism can prove no more than is involved in the premises". ${ }^{9}$

So, in both types of reasoning there is a semantic relation between the premise and the conclusion: both are analytic, not only material deduction. This means too, and thinking specially in formal deduction, that the 'dogma of the form' or the 'substitutability dogma' must be abandoned: there aren't truths and correct deductions arising from the "structure" of propositions and, although the meaning of the non-logical terms is irrelevant in formal deduction, the meaning of the so called 'logical terms' (like the meaning of the propositional connectives, which is given by the truth tables) is not irrelevant.

Concerning this alleged boundary between formal and material deductions, Tarski, for example, says that:

“(...) no objective grounds are known to me which permit us to draw a sharp boundary between the two groups of terms. It seems to be possible to include among logical terms some which are usually regarded by logicians as extra-logical without running into consequences which stand in sharp contrast to ordinary usage. In the extreme case we could regard all terms of the language as logical. The con-

$\overline{9 \quad \text { Stuart Mill, 1 }} 974,183$. 
cept of formal consequence would then coincide with that of material consequence". ${ }^{10}$

And just as logical terms are not substitutable in formal deduction (they are constants), there are substitutable terms (variables) in material deduction: for instance, when we infer that $x$ is a belief from $x$ being (a case of) propositional knowledge, we do not take into account what $x$ is.

Analyticity exists too when the identity between what is said in the premise and what is said in the conclusion is total, for instance, in inferences that correspond to the so called 'logical equivalences'. Here's an example:

C. Socrates is short and Plato is tall

$\therefore$ it's not the case that Socrates isn't short or Plato isn' $t$ tall.

Under what conditions is the premise true? The premise is true if and only if Socrates is short and Plato is tall. And under what conditions is the conclusion true? The conclusion is true if and only if Socrates is short and Plato is tall. The premise and the conclusion have exactly the same truth conditions (meaning). The truth tables of the conjunction and of the negation of a disjunction of negations make that clear:

Conjuntion

Negation of a disjunction of negations

\begin{tabular}{|c|c|c|}
\hline $\mathbf{p} \mathbf{q}$ & $(\mathbf{p} \wedge \mathbf{q})$ & $\neg(\neg \mathbf{p} \vee \neg \mathbf{q})$ \\
\hline $\mathrm{VV}$ & $\mathrm{V}$ & $\mathrm{V} \mathrm{FFF}$ \\
\hline $\mathrm{VF}$ & $\mathrm{F}$ & $\mathrm{F} \mathrm{FVV}$ \\
\hline $\mathrm{FV}$ & $\mathrm{F}$ & $\mathrm{F} \mathrm{VVF}$ \\
\hline $\mathrm{FF}$ & $\mathrm{F}$ & $\mathrm{F} \mathrm{VVV}$ \\
\hline
\end{tabular}

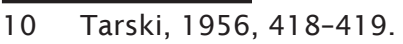


The common synonyms of ordinary language also provide cases of absolute identity between premise and conclusion:

D. $x$ is an abstract of my paper

$\therefore x$ is a summary of my paper.

As in reasoning corresponding to logical equivalences, in this type of reasoning one is only replacing one term or expression with another with the same meaning: in the first case (an instantiation of one of the De Morgan's Laws), the occurrence of 'and' is replaced by 'not (not ... or not ...)'; in the second case, one is not dealing with the meaning of propositional connectives but with the meaning of "normal" terms. In any case, one is not inferring something new but only proceeding to a linguistic substitution. ${ }^{11}$

What should we conclude? According to the assumed definition, a valid deduction is the one where the truth of the conclusion derives with certainty (or warrant) from the truth of the premise(s). We saw that such cases correspond to the ones where the conclusion says nothing different from the premises: like Mill asserts, there is no progress from the known to the unknown. In other words: there is no novelty.

It seems that there is an intrinsic relation: if there is certainty (or warrant), than there is no novelty. This is the traditional understanding of deduction: according to it, for the conclusion to be correctly derived from the premise(s), one must not say more in the conclusion than what is already said in the premise(s); or, in order to get warrant, one must give up novelty.

On the other hand, one may equally say that such cases are, at the outset, all the cases of lack of novelty and that they all correspond to the presence of warrant. Then, if one does not have novelty, one gains from this warrant: novelty and warrant cannot both inexist.

If novelty and warrant are incompatible, then whenever we have novelty we lose warrant: that's exactly what seems to happen in abductive reasoning. And, if warrant and novelty cannot both inexist, then too when-

11 "(...) in studying the laws of signs, we are in effect studying the manifested laws of reasoning", Boole, 1854, 24. 
ever we do not have warrant, we gain from this novelty: again, that's what seems to happen in abductive reasoning.

\section{Synthetic Reasoning}

Let's assume the following definition of synthetic reasoning: what is said in the conclusion is different from what is said in the premise(s) there is not identity. Causal reasoning fits perfectly into this definition: that is why Hume said that all reasonings concerning matter of fact (and not relations of ideas) seem to be founded on the relation of cause and effect. And one might say that causal reasoning lacks warrant because it is synthetic. Therefore, causal reasoning would be an instance of inductive reasoning.

To confirm this suspicion, let's look at an example of synthetic reasoning:

E. The floor is wet in I

$\therefore$ It is raining in $I$.

This is reasoning from the effect to the cause. Hence, the conclusion says something different form the premise: in order for something to cause another thing, it must be different from the thing it causes. No doubt, there is novelty. Besides that, this is not a deduction: someone could have thrown a bucket of water in order for the floor to be wet. Instead, it is a case of Peirce's abductive reasoning, still a kind of induction. According to Peirce in Pragmatism and Induction - Abduction and Perceptual Judgments:

"The surprising fact, $C$, is observed. But if A were true,

$C$ would be a matter of course. Hence, there is reason to suspect that $A$ is true". ${ }^{12}$

In abductive reasoning, the gain of novelty (synthetic reasoning) comes with the lack of warrant (induction). Because the conclusion says something different from the premise(s) - if there is progress from the known to the unknown -, there is not any certainty that the conclusion derives

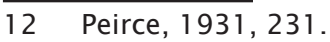


from the premise(s) - one can only infer the probable explanation of some fact.

Philosophical tradition agrees with this in general. Like Francis Bacon said in the Novum Organum:

"There are, and can be, only two ways to investigate and discover truth. The one leaps from sense and particulars to the most general axioms, and from these principles and their settled truth, determines and discovers intermediate axioms; this is the current way. The other elicits axioms from sense and particulars, rising in a gradual and unbroken ascent to arrive at last at the most general axioms; this is the true way, but it has not been tried". ${ }^{13}$

Although Bacon was thinking in traditional induction, believing it to be a case of synthetic reasoning (which is not, as I will try to show), the point is that he is expressing the traditional view according to, once one has novelty, one loses warrant.

As we have seen, the incompatibility principle holds true for abduction. And the negative incompatibility principle holds true too: the lack of warrant (induction) comes with the gain of novelty (synthetic reasoning).

But does the incompatibility principle hold true for every case of causal reasoning? Is every causal reasoning inductive? According to the traditional view, yes. But I think that the traditional view is wrong. And it rests on a mistake: the confusion between inferring the cause of an event and inferring the causal relation itself. As I have said, that mistake goes way back Hume; is certainly present in Copi, Cohen and McMahon's paper. After including causal reasoning in the list of types of induction, the authors distinguish between the establishment of the (supposed) relation between cause and effect and the reasoning based on those relations. This a crucial difference: the establishment of the relation between cause and effect can be inductive without the reasoning based on those

13 Bacon, 2000, 36. 
relations being inductive too. But that is a corollary the authors did not take into account.

Let's assume the truth of the following proposition: if one gets close to a flame, then one will feel heat (Hume's example). The next two propositions can both be true: i) there is no warrant that the proposition 'if one gets close to a flame, then one will feel heat' is true; ii) the prediction that when one gets close to a flame, one will feel heat is correct (because the proposition 'if one gets close to a flame, then one will feel heat' is true).

Tradition hasn 't paid much attention to this distinction, ${ }^{14}$ which is grounded in another one: one thing is the internal certainty of any causal relation (just for being a causal relation, any causal relation has certainty); another thing is to warrant the existence of causal relations, which is an external certainty. Or, which is the same, we must distinguish the meaning of the word 'causality' from the existence of causality. By definition, all causal relations are certain: this is why Kant spoke of synthetic a priori judgments - if a causal relation exists, than certainty (or warrant) exists. However, the knowledge of those relations may not be certain: Hume drove our attention to this fact - we may never be certain of the existence of causal relations.

Hence, one thing is to warrant the truth, and another thing is the truth of that warrant. For instance: the application of a force in a ball warrants that the ball will move but we can still ask what warrants that, if we apply a force in a ball, then the ball will move - here the warrant is about the truth of the all conditional, not about the truth of the consequent (that the ball will move).

So, following Kant, one may say that, by definition, causal relations are warrant nexus: the cause produces certainly the effect. Therefore, reasoning from one to another is justified, that is, has warrant. But, following Hume, one may say that there is no certainty of the existence of those causal relations. Therefore, reasoning ending in a causal judgment isn't certain.

$\overline{14}$ With exception, for example, of the work of Michael Scriven, like in Scriven, 1962. 
Two different kinds of reasoning are involved:

F. It is raining

$\therefore$ The floor is wet.

G. It is raining and the floor is wet

$\therefore$ Since it's raining, than the floor is wet.

In the first case, there is warrant (if there is a valid law connecting premise and conclusion: if it 's true that raining causes the floor being wet). In the second case, there is not warrant but only probability (the premise does not prove that 'if it's raining, than the floor is wet' is true). One may lack certainty about the truth of the proposition 'if it's raining, than the floor is wet' but, nevertheless, if that's true, than it is certain that, if it's raining, than the floor is wet.

Therefore, the argument which supports the traditional view (according to which all cases of causal reasoning are of the inductive type) - if the knowledge of causal relations lacks warrant, than causal reasoning lacks warrant - is fallacious, reason why the possibility of deductive causal reasoning is still open. And, by analogy with Kantian synthetic a priori judgements, I argue that synthetic deductions are actually conceivable. Analytic deductions (the analogous of analytic judgements) and synthetic inductions (the analogous of synthetic judgments) are not the only types of reasoning conceivable.

Analytic judgements express certainty (necessity, in Kant's words), since the predicate is contained in the subject - in other words, subject-predicate sentences are analytical when the predicate belongs to the meaning of the subject. They have a priori source: we know that a case of propositional knowledge is a belief without having experience of the (extra-linguistic) world.

Synthetic judgements express a relation in which the predicate goes beyond the subject - in other words, subject-predicate sentences are synthetic when the predicate doesn 't belong to the meaning of the subject. Their source is a posteriori: we know that Socrates is mortal only if we have experience of the (extra-linguistic) world. 
In Kant's own words:

"In all judgments in which the relation of a subject to the predicate is thought (if I only consider affirmative judgments, since the application to negative ones is easy) this relation is possible in two different ways. Either the predicative $B$ belongs to the subject $A$ as something that is (covertly) contained in this concept $A$; or $B$ lies entirely outside the concept $A$, thought to be sure it stands in connection with it. In the first case I call the judgment analytic, in the second synthetic. Analytic judgments (affirmative ones) are thus in which the connection of the predicate is thought through identity, but those in which this connection is thought without identity are to be called synthetic judgments. One could also call the former judgments of clarification, and the later judgments of amplification". ${ }^{15}$

But one kind of judgment doesn't fall under the analytic-synthetic distinction: causal judgments. On the one hand, they have novelty, since cause and effect are different events. On the other hand, they have certainty, since cause and effect are linked in a deterministic way.

In order to qualify them, Kant conceived a third category of judgments: the synthetic a priori judgments - synthetic judgments with certainty. Besides causal judgments, Kant thought that other kind of judgments (like the ones from mathematics) where synthetic a priori too. I will not consider them as far there is controversy over whether Kant is right or not.

This third category of judgments are introduced by Kant in a particular context: Kant wants to save Science (namely Physics and more specifically Newtonian Mechanics) from the merciless attack undertook by Hume. Kant's Critique of Pure Reason was thought and developed to explain the possibility of synthetic a priori judgments, or, same thing (at least, to Kant), to explain the possibility of scientific knowledge - the one who aims to discover true causal relations and not mere constant

15 Kant, 1998, 141. 
conjunctions. Hume argued that it is impossible to warrant the existence of cause and effect relations: neither reason nor the senses are capable of showing us that successions of events are linked in a causal nexus.

The scientific world-view compels us to interpretations like the following one:

Succession of events: it's raining and the floor is wet.

Correct interpretation: since it's raining then the floor is wet.

According to this view, it is not only true that the floor gets wet after the rain - it gets wet because it's raining. The use of the scientific method ensures the correction of such interpretation. But Hume denies the possibility of such warrant. To him, cause effect links are nothing but a psychological association grounded on the habit of observing one event succeeding another event.

The Humean world-view is different from the scientific one:

Succession of events: it's raining and the floor is wet.

Psychological interpretation: since it's raining, then the floor is wet.

Correct interpretation: it's raining and the floor is wet.

As Hume says in his Treatise of Human Nature:

"Thus we remember to have seen that species of object we call flame, and to have felt that species of sensation we call heat. We likewise call to mind their constant conjunction in all past instances. Without any farther ceremony, we call the one cause and the other effect, and infer the existence of the one from that of the other. (...)"16

Hume is warning us that the kind of reasoning we make when we go from regular and diachronic conjunctions to causal relations is an inductive one, no matter how probable the conclusion is. The problem, as I said before, is that from the impossibility of warrant regarding the knowledge of causal relations, Hume - if not him, those who followed him - asserts the impossibility of warrant regarding causal reasoning. This is a "mortal leap": causal relations may exist (and, therefore, scientific knowledge is

16 Hume, $1975,87$. 
possible) even though no one can ever prove that. The scientific worldview is not incompatible with the Humean world-view.

\section{Synthetic Deductive Reasoning}

Certainty or internal warrant is all what is required in order for deductive reasoning to exist. By definition, causal relations have that certainty: hence, deductive causal reasoning is conceivable as much as causal relations are conceivable. Besides that, deductive causal reasoning has the advantage of having novelty, because, as all causal reasoning, deductive causal reasoning is synthetic reasoning. Thus, the incompatibility between warrant and novelty proofs to be a false one: in deductive causal reasoning, one reasons with certainty, although the conclusion is not included in the meaning of the premise - one reasons with novelty too. Hence one gets what a deduction ought to be: safe acquisition of new knowledge.

Kant showed us that not all warrant judgments are analytic. Now one can also say that warrant reasoning is not necessarily analytic. So, on the one hand, one can reason with or without warrant - deduction versus induction -, and, on the other hand, one can reason with or without novelty - synthetic versus analytic reasoning.

I must add that the problem of induction posed by Hume is of no matter to those who use the causal inferential rule; it concerns to those who must know and proof it: it's a problem Science must handle - the problem of the scientific method. Those who use the rule only have to presuppose its validity (the truth of the causal judgment).

But I must present three restrictions to what I have said so far concerning synthetic deduction.

First restriction: causal reasoning is really only from effect to cause. Although the phrase 'causal reasoning' is usually applied in a broad sense, when one derives the effect from the cause one is making instead a prediction. To reason is to know some truth by means of the knowledge of another truth; but one can predict an effect that has not happened yet and, therefore, whose truth is not yet available for knowledge. Prediction is pre-knowledge, to anticipate the knowledge of some truth. 
One can summarize this restriction in a slogan: causation and implication are the "two sides of the same coin". If a prediction is made based on the causation side of the coin:

$F$. It is raining in $I$

$\therefore$ The floor is wet in I

then the deduction based on the implication side of the coin goes like this:

H. The floor is wet in I

$\therefore$ It is raining in $I$.

But in this example still doesn't exist deductive reasoning: again, someone could have thrown a bucket of water. A problem arises: both types of causal reasoning, causal deduction and abduction, are based on causal relations - in what do they differ from each other? This is why we need a second restriction: one can only reason from effect to cause with certainty if the cause is a necessary condition, not a sufficient one, of the effect.

Therefore, we must change the example:

I. Sofia had a good grade on her exam

$\therefore$ Sofia studied for the exam.

Since Sofia's studying for the exam is (causally) necessary for her to have a good grade (let's supposed that), the inference from this last fact to the first one is secure, i.e, Sofia having a good grade on her exam is (logically) sufficient for her to have studied for the exam. If the causal condition is a sufficient one, then the logical condition is only a necessary one.

Finally, the third restriction: the causation side of the coin must be counterfactual (or virtual) in order for the implication side of the coin to be actual. This restriction arises from the imperative of non-contradiction. Let's suppose that it is actually false that Sofia studied for the exam: then (and this is a causal 'then') it is actually false that Sofia had a good grade. But, then, one cannot deduce the actual truth that Sofia studied for the exam from the actual truth that she had a good grade, since it would be true and false in the actual world that she had a good grade and, too, that she studied for the exam. One can only deduce 
that Sofia studied for the exam from her having a good grade on it if it would be false that she had a good grade on her exam hadn't she study for it.

\section{Analytic Inductive Reasoning}

So far, I have presented a counter-example to the universal application of the incompatibility principle: synthetic deductive reasoning. And how about the negative incompatibility principle? Is analytic inductive reasoning conceivable? At first glance, the concept of analytic induction is strange: how can there be no guarantee (it would be an induction) nor novelty (it would be analytic)?

Let's assume the following definition of strong inductive reasoning: the truth of the conclusion derives with probability from the truth of the premise(s). By (this) definition, induction lacks certainty. Let's look at a couple of examples of (more or less) strong inductive reasoning according to the tradition:

J. Parmenides (who is a man) is mortal and Pythagoras (who is a man) is mortal and Socrates (who is a man) is mortal and Plato (who is a man) is mortal

$\therefore$ All men are mortal

K. $x$ is a belief $+x$ is true

$\therefore x$ is (a case of) propositional knowledge

The first type of reasoning is induction by generalization and the second type is induction by analogy. Now let's substitute the sentences contained in the conclusion for what they mean:

J. Parmenides (who is a man) is mortal and Pythagoras (who is a man) is mortal and Socrates (who is a man) is mortal and Plato (who is a man) is mortal

$\therefore$ Parmenides (who is a man) is mortal and Pythagoras (who is a man) is mortal andSocrates (who is a man) is mortal and Plato (who is a man) is mortal and etc.

K. $x$ is a belief $+x$ is true

$\therefore x$ is a belief $+x$ is true + etc. 
'Etc.', again, appears as a representative of what's missing in order for the meaning of the sentences to be complete. The examples seem to certify that something is being added to the premise and, therefore, that the loss of warrant entails the gain of novelty. But that "something being added" is merely apparent: if one adds to the premise what is missing in order for it to warrant the conclusion, then one gets exactly the same as the conclusion:

L. $x$ is a belief $+x$ is true + etc.

$\therefore x$ is a belief $+x$ is true + etc.

This shows that traditional induction aims identity: despite being inductive, it is, too, analytic. And there is already some identity: excluding what is represented by 'etc.', premise and conclusion are identical. That is something that never happens with synthetic inductive reasoning: the premise is not even partially identical with the conclusion and, if completed in order to warrant the conclusion, it does not became totally identical with it.

Being a case of analytic reasoning, traditional induction constitutes a counter-example to the universal application of the negative incompatibility principle. One can reason without warrant and too without novelty.

\section{Criminal and Medical Reasoning}

The results l've presented have many practical consequences, since causal reasoning is of great importance to some activities, most notorious criminal investigation and medical practice. In both cases we start from evidence in order to reach, in the first case, the author of the crime and, in the second case, the disease. And in both cases we need to be sure - to not to condemn innocents and not to treat the wrong disease. Again, in both cases science comes to rescue, providing the knowledge of the relevant causal relations.

In those activities there is real deductive reasoning being made, although Humean skepticism concerning the justification of the beliefs in which those deductions are based is a problem one cannot underestimate. 
Atocha Aliseda, in her book Abductive Reasoning - Logical Investigations into Discovery and Explanation, for example, makes special reference to the importance of causal reasoning:

"Broadly speaking, abduction is a reasoning process invoked to explain a puzzling observation. A typical example is a practical competence like medical diagnosis. When a doctor observes a symptom in a patient, she hypothesizes about its possible causes, based on her knowledge of the causal relations between diseases and symptoms. This is a practical setting. Abduction also occurs in more theoretical scientific contexts. For instance, it has been claimed [Han61],[CP, 2.623] that when Kepler discovered that Mars had an elliptical orbit, his reasoning was abductive. But, abduction may also be found in our day-to-day common sense reasoning. If we wake up, and the lawn is wet, we might explain this observation by assuming that it must have rained, or by assuming that the sprinklers had been on. Abduction is thinking from evidence to explanation, a type of reasoning characteristic of many different situations with incomplete information". ${ }^{17}$

It is then disappointing that the author restricts causal reasoning (the search for explanations) to abduction. She makes a good point by invoking, for example, the process of medical diagnosis, in which, based on the knowledge of the causal relation between disease and symptom, the doctor infers the first from the second. But, by reducing causal reasoning to abduction, she is making the same mistake tradition made, i.e., to take the discovery of the cause to be the discovery of the causal relation. Besides that, Aliseda's book has its own problems: because Aliseda puts abduction at the heart of scientific discovery, she also takes the discovery of the causal relations to be the discovery of the cause. In both cases, the distinction between the two types of discovery is not being made, with the implicit acceptance of the incompatibility principle.

17 Aliseda, 2006, 41. 


\section{References}

Aliseda, Atocha. Abductive Reasoning - Logical Investigations into Discovery and Explanation. Dordrecht: Springer, 2006.

Aristotle. The Categories/On Interpretation/Prior Analytics. Edited by Harold Cook and Hugh Tredennick. London: William Heinemann Ltd, 1970.

Bacon, Francis. The New Organon. Edited by Lisa Jardine and Michael Silverthorne. Cambridge: Cambridge University Press, 2000.

Boole, George. An Investigation of the Laws of Thought. London: Walton and Maberly, 1854.

Copi, Irving, Cohen, Carl and McMahon, Kenneth. "Causal Reasoning". In Introduction to Logic, edited by Copi, Irving, Cohen, Carl and McMahon, Kenneth. Edinburgh: Pearson New International, 2014.

Downing, P.B.. "Conditionals, Impossibilities and Material Implications". Analysis, Vol. 35, No. 3 (1975): 84-91.

Edgington, Dorothy, "Indicative Conditionals", The Stanford Encyclopedia of Philosophy (Winter 2014 Edition), Edward N. Zalta (ed.), URL $=\langle$ http://plato.stanford.edu/archives/win2014/entries/conditionals/ $>$

Frege, Gottlob. Collected Papers on Mathematics, Logic and Philosophy. Edited by Brian McGuiness. Oxford: Basil Blackwell Publisher, 1984.

Hausman, Alan, Kahane, Howard and Tidman, Paul. Logic and Philosophy - A Modern Introduction. Boston: Wadsworth, Cengage Learning, 2010.

Hempel, C.G.. "Aspects of Scientific Explanation". In Aspects of Scientific Explanation and Other Essays in the Philosophy of Science. New York: The Free Press, 1965, 331-496.

Hempel, C.G. and Oppenheim, P.. "Studies in the Logic of Explanation". Philosophy of Science 62, 1948, 135-175.

Horn, Laurence R., "Contradiction", The Stanford Encyclopedia of Philosophy (Spring 2014 Edition), Edward N. Zalta (ed.), URL $=<$ http://plato. stanford.edu/archives/spr2014/entries/contradiction/>.

Hume, David. A Treatise of Human Nature. Edited by L.A. Selby-Bigge. Oxford: Clarendon Press, 1975. 
An Enquiry Concerning Human Understanding. Edited by Peter Millican. Oxford: Oxford University Press, 2007.

Kant, Immanuel. Critique of Pure Reason. Translated and Edited by Paul Guyer and Allen Wood. Cambridge: Cambridge University Press, 1998.

Lewis, C.I.. A Survey of Symbolic Logic. Berkeley: University of California Press, 1918.

MacColl, H.. "Symbolical Reasoning". Mind, Vol. 5, N. ${ }^{\circ} 17$ (1880): 45-60.

---- "Symbolical Reasoning (II)". Mind, Vol. 6, N. ${ }^{\circ} 24$ (1 897): 493-510. "Symbolical Reasoning (III)". Mind, Vol. 9, N. 33 (1900): 75-84. "Symbolical Reasoning (IV)". Mind. Vol. 11, N. ${ }^{\circ} 43$ (1902): 352-368.

----- "Symbolical Reasoning (V)". Mind. Vol. 12, N. ${ }^{\circ} 4$ (1903): 355-364.

----- "Symbolical Reasoning (VI)". Mind. Vol. 14, N. ${ }^{\circ} 53$ (1905): 74-81.

---- "Symbolical Reasoning (VII)". Mind. Vol. 14, N.o 55 (1905): 390-397. 504-518.

"Symbolical Reasoning (VIII)". Mind. Vol. 15, N. ${ }^{\circ} 60$ (1906):

Symbolic Logic and its Applications. London: Longmans, Green, and Co., 1906.

Peirce, Charles Sanders. "Pragmatism as the Logic of Abduction". In The Essential Peirce - Vol. 2. Edited by the Peirce Edition Project. Indiana: Indiana University Press, 1931.

Priest, Graham. Lógica. Tradução de Célia Teixeira. Lisboa: Temas e Debates, 2002.

Putnam, Hilary. Philosophy of Logic. New York: Routledge, 2010.

Sanford, David. If P, Then Q: Conditionals and the Foundations of Reasoning. London: Routledge, 1989.

Scriven, Michael. Explanations, Predictions and Laws. Minneapolis: University of Minnesota Press, 1962.

Stuart Mill, John. "A System of Logic - Ratiocinative and Inductive". In Collected Works of John Stuart Mill - Vol. VII. Toronto: Toronto University Press, 1974. 
Tarski, Alfred. "On the Concept of Logical Consequence". In Logic, Semantics, Mathematics - Papers from 1923 to 1938. Translated by J.H. Woodger. Oxford: Clarendon Press, 1956.

Von Wright, Georg Henrik. An Essay in Modal Logic. Amsterdam: North-Holland Publishing Company, 1951.

Whitehead, A.N., and Russell, Bertrand. Principia Mathematica. Cambridge: Cambridge University Press, 1997.

Wittgenstein, Ludwig. Tratado Lógico-Filosófico/Investigações Lógicas. Tradução de M.S. Lourenço. Lisboa: Fundação Calouste Gulbenkian, 2011.

Zilhão, António. Lógica - 40 Lições de Lógica Elementar. Lisboa: Edições Colibri, 2001. 